\title{
Hydrothermal Synthesis of Three-dimensional Butterfly-like Ni Architectures as Microwave Absorbers
}

\author{
Yuping Sun ${ }^{a, b}$, Niandu Wu ${ }^{b, c}$, Caiyun Cui ${ }^{b, c}$, Siu Wing Or ${ }^{b}$, Xianguo Liu ${ }^{c *}$ \\ ${ }^{a}$ Center for Engineering practice and Innovation Education, Anhui University of Technology - AHUT, \\ Maanshan 243032, PR China \\ ${ }^{b}$ Department of Electrical Engineering, The Hong Kong Polytechnic University - PolyU, \\ Hung Hom, Kowloon, Hong Kong, China \\ cAnhui Key Laboratory of Metal Materials and Processing, School of Materials Science and \\ Engineering, Anhui University of Technology - AHUT, Maanshan 243002, PR China
}

Received: August 14, 2015; Revised: September 12, 2015

\begin{abstract}
Three-dimensional butterfly-like $\mathrm{Ni}$ architectures were fabricated by a surfactant-assisted hydrothermal method. The Ni architectures, with lengths of about $20 \mu \mathrm{m}$ and widths of 4-6 $\mu \mathrm{m}$, were assembled from tens of Ni nanorods with the averaged diameter of about $200 \mathrm{~nm}$. The Ni nanorods were coated by oxide shells. The saturation magnetization of the Ni architectures was found to be $66.2 \mathrm{emu} / \mathrm{g}$. The magnetic loss in the $\mathrm{Ni}$ architectures was mainly caused by the natural resonance, while the dielectric relaxation loss was originated from the interfacial relaxation between the oxide shells and the Ni nanorods in addition to the size distribution and morphology of the Ni architectures. Absorbers with a thickness of $2.1 \mathrm{~mm}$ exhibited an optimal reflection loss (RL) value of $-38.9 \mathrm{~dB}$ at $12.8 \mathrm{GHz}$. RL values exceeding $-20 \mathrm{~dB}$ in the $8.0-17.8 \mathrm{GHz}$ range were obtained by choosing absorber thicknesses between 1.5 and $3.2 \mathrm{~mm}$. A quarter-wavelength cancellation model was used to explain the observed thickness dependence of RL peak frequency.
\end{abstract}

Keywords: nanomaterials, electromagnetic properties, magnetic materials

\section{Introduction}

The development of electromagnetic (EM) wave absorbers has been a recent focus towards solving the serious EM pollution arising from the fast-growing generation and applications of electronic devices ${ }^{1-5}$. Among various potential candidates for EM wave absorbers, the one in the form of magnetic nanoparticles core/ dielectric shell-structured nanocapsules, such as $\mathrm{Ni} / \mathrm{ZnO}, \mathrm{FeNi}_{3} / \mathrm{SiO}_{2}, \mathrm{Ni} / \mathrm{PANI}, \mathrm{Ni} / \mathrm{C}$, and $\mathrm{Ni} / \mathrm{Ni}_{2} \mathrm{O}_{3}$ nanocapsules, has been of particular interest in recent years owing to its tailorable properties in conjunction with smaller sizes and weights ${ }^{6-10}$. An essence of determining the EM wave absorbing properties of the nanocapsules is to obtain a balance between the permeability of the magnetic nanoparticle cores with the permittivity of the dielectric shells ${ }^{11}$. However, the generally low permeability $(\sim 1)$ intrinsic in the magnetic nanoparticle cores at gigahertz frequencies, has constrained the strong absorption in some limited frequency range with large variations with absorber thickness.

More recently, improved high-frequency permeabilities have been observed in magnetic nanomaterials with different morphologies, including polyhedral Fe nanocomposites ${ }^{11}$, $\mathrm{FeNi}_{3}$ nanorods ${ }^{12}$, dumbbell-like $\mathrm{Fe}_{3} \mathrm{O}_{4}$-Au nanoparticles ${ }^{5}$, hierarchical branch- and flower-like Ni microcrystals ${ }^{13}$ and $\mathrm{Fe}$ nanoflakes ${ }^{14}$. The improvement in high-frequency permeabilities has been attributed to an increased in their magnetic shape anisotropy. In fact, $\mathrm{Ni}$ is an important soft magnetic metal with a large saturation magnetization $\left(M_{S}\right)$,

*e-mail: liuxianguohugh@gmail.com a high permeability, a high anti-oxidation ability, and low energy losses. In this paper, we report the synthesis of three-dimensional (3D) butterfly-like $\mathrm{Ni}$ architectures by a surfactant-assisted hydrothermal method as well as the microstructure, magnetic, dielectric, and EM wave absorbing properties of the architecture.

\section{Experimental Procedure}

All chemicals used were of analytical grade and were used as received. In a typical synthesis, $\mathrm{NiCl}_{2} 6 \mathrm{H}_{2} \mathrm{O}$ ( $0.476 \mathrm{~g}, 2 \mathrm{mmol})$, sodium dodecyl benzenesulfonate (SDBS) $(0.232 \mathrm{~g}, 1 \mathrm{mmol})$ and $\mathrm{NaOH}(1.6 \mathrm{~g}, 40 \mathrm{mmol})$ were dissolved in deionized water $(40 \mathrm{~mL})$ under constant stirring, resulting in a precursor green suspension. To prepare the proposed 3D butterfly-like $\mathrm{Ni}$ architectures, the precursor green suspension was transferred into a $50-\mathrm{mL}$ Teflon-lined stainless steel autoclave. The autoclave was sealed and heated at $100{ }^{\circ} \mathrm{C}$ for $15 \mathrm{~h}$ and then cooled to room temperature naturally. The products obtained after the hydrothermal treatment were centrifuged, washed with distilled water and ethanol several times and finally dried in vacuum at $60{ }^{\circ} \mathrm{C}$ for $4 \mathrm{~h}$.

The composition and phase purity of the as-prepared products were examined by an X-ray diffractometer (XRD, Brucker D8 Advance, Germany) at $40 \mathrm{kV}$ voltage and $50 \mathrm{~mA}$ current and with $\mathrm{CuK}_{\alpha}$ radiation $(\lambda=1.5418 \AA)$. The morphologies of the products were captured using a scanning electron microscope (SEM, JEOL-6300F, Japan) at 
an acceleration voltage of $20 \mathrm{kV}$ and a transmission electron microscope (TEM, JEOL-2010F, Japan) at an acceleration voltage of $200 \mathrm{kV}$. The magnetization curves were measured using a vibration sample magnetometer (VSM 7407, China).

Paraffin-bonded Ni architecture composites were prepared by uniformly mixing $40 \mathrm{wt} . \% \mathrm{Ni}$ architectures in the paraffin matrix and by pressing the mixture into cylinder-shaped compacts. More details can be found elsewhere ${ }^{6,11,12}$. The prepared compacts were cut into toroidal samples with $7.00 \mathrm{~mm}$ outer diameter and $3.04 \mathrm{~mm}$ inner diameter. The EM parameters of the toroidal samples were evaluated in the frequency range of 2-18 GHz, using a vector network analyzer (Agilent N5244A, USA) in conjunction with a coaxial method in transverse EM mode. The vector network analyzer was calibrated for the full two-port measurement of reflection and transmission at each port. The complex permittivity $\left(\varepsilon_{r}=\varepsilon^{\prime}-j \varepsilon^{\prime \prime}\right)$ and complex permeability $\left(\mu_{r}=\mu^{\prime}-j \mu^{\prime \prime}\right)$ were calculated from S-parameters tested by the vector network analyzer using the simulation program of Reflection/Transmission Nicolson-Ross model ${ }^{11}$.

\section{Results and Discussion}

The XRD pattern in Figure 1 shows the phase components of the as-prepared products. All XRD peaks can be indexed to the single-phase face-centered cubic $\mathrm{Ni}$ and are consistent with the standard card JCPDS No. 04-0850 (space group $F m \overline{3} m$; $\mathrm{a}=3.523 \AA$ ). No reflections for oxides are found, which can be ascribed to the anti-oxidation nature of $\mathrm{Ni}$. The average grain size of $\mathrm{Ni}$ is estimated to be $24.8 \mathrm{~nm}$ by using the reflection peak of (111) and Debye-Scherrer's relation. The inset of Figure 1 shows the hysteresis loop of the products at $295 \mathrm{~K}$. The $M_{S}$ of the products reaches $66.2 \mathrm{emu} / \mathrm{g}$, which is bigger than that of $\mathrm{Ni} / \mathrm{C}$ nanocapsules ${ }^{9}$, but, is smaller than that of bulk nickel because of the size effect ${ }^{9}$.

Figure 2 shows the typical morphology of the products with different magnifications. The SEM image in Figure 2a implies that the products are butterfly-like architectures with a length of about $20 \mu \mathrm{m}$ and a width of 4-6 $\mu \mathrm{m}$. The magnified SEM image in Figure $2 \mathrm{~b}$ shows that the butterfly-like architectures

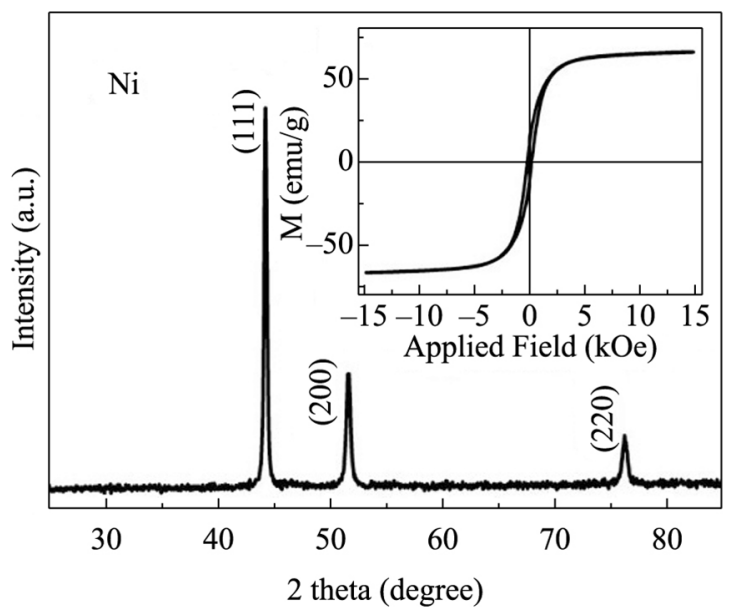

Figure 1. XRD pattern of the products. The inset shows hysteresis loop of products at $295 \mathrm{~K}$. are composed of tens of order-attached nanorods, each with averaged length of about $20 \mu \mathrm{m}$ and the averaged diameter of about $200 \mathrm{~nm}$. It is also observed that the nanorods are attached together in the middle part of an individual butterfly-like architecture and become curly and separated at the two ends with a symmetric character. The TEM image of the products in Figure 2c further confirms our observation in Figure 2a that the Ni architectures are self-assembled by nanorods. A typical HRTEM image of one nanorod in Figure 2d, clearly indicates that the d-spacing of $0.2 \mathrm{~nm}$ corresponds to the lattice fringe $\{111\}$ of $\mathrm{Ni}$. In addition, few amorphous oxides are seen on the surface of nanorods as a result of the high surface energy from small size effect ${ }^{11}$. The formation if the architectures possibly consists of four steps: (1) Formation of Ni crystal nuclei. (2) Ni nuclei grow into Ni nanoribbons. (3) The Ni nanoribbons self-assemble into Ni nanorods through an oriented attachment mechanism. (4) Ni nanorods attach orderly and assemble into butterfly-like architecture.

The $\varepsilon_{r}$ and $\mu_{r}$ of the paraffin-based composites are fundamental physical quantities in determining the microwave absorbing properties ${ }^{15}$. Figure 3 a shows the frequency $(f)$ dependence of $\varepsilon_{r}$ for the paraffin-bonded Ni architecture composites. Both the real part $\left(\varepsilon^{\prime}\right)$ and imaginary part $\left(\varepsilon^{\prime \prime}\right)$ display a similar decreasing trend with increasing frequency from 2 to $18 \mathrm{GHz}$. The results may be caused by an increased lagging in the dipole polarization response with respect to the electric field change at higher frequencies ${ }^{16}$. The maximum/minimum values can be found below/above the resonance frequency in the $\varepsilon^{\prime}$ curve $e^{6}$. Accordingly, one peak is observed in the $\varepsilon$ " curve near the resonance frequency. The resonance frequency of $\varepsilon$ in the current frequency range is $14.8 \mathrm{GHz}$. From the plot of $\varepsilon^{\prime}$ versus $\varepsilon$ ", the Cole-Cole semicircle, as shown in Figure 3c. the composites present a clear segment of one semicircle at high frequencies and a linear curve at relatively low frequencies. The presence of one semicircle at high frequencies suggests that there is a Debye dielectric relaxation process due to the dielectric relaxation of the interfacial relaxation between the oxide shells and the Ni nanorods ${ }^{16}$. The dielectric relaxation is significant for the enhancement of the microwave absorption in the $\mathrm{Ni}$ architectures. Besides the dielectric relaxation process, other dielectric mechanisms such as resistance loss and defects may contribute to the permittivity dispersion, leading to the linear curve at relatively lower frequencies ${ }^{17,18}$. Following the effective-medium theory of three-phase inclusions ${ }^{19}$, the permittivity of the composites seems to originate from the special geometric structures of the Ni architecture inclusions, the intrinsic permittivity of each component in the composites (Ni, oxides, and paraffin), and the dispersion (volume fraction) of the composites.

From Figure $3 \mathrm{~b}$, the real part $\left(\mu^{\prime}\right)$ of $\mu_{r}$ decreases from 1.42 to 1.04 with increasing frequency in frequency range of 2-5.6 and becomes almost independent of frequency for frequencies up to $18 \mathrm{GHz}$. Meanwhile, the imaginary part ( $\left.\mu^{\prime \prime}\right)$ of $\mu_{r}$ has a resonance peak at $4.0 \mathrm{GHz}$ with a broad resonance band covering 3-11 GHz. The resonance frequency at $4.0 \mathrm{GHz}$ is due to the large anisotropy energy of $\mathrm{Ni}$ architectures ${ }^{6,9}$. The anisotropy energy of particles of small sizes, especially in the nanometer scale, may be remarkably increased due to the size effect-induced shape/surface anisotropy ${ }^{6,9}$. The broad 


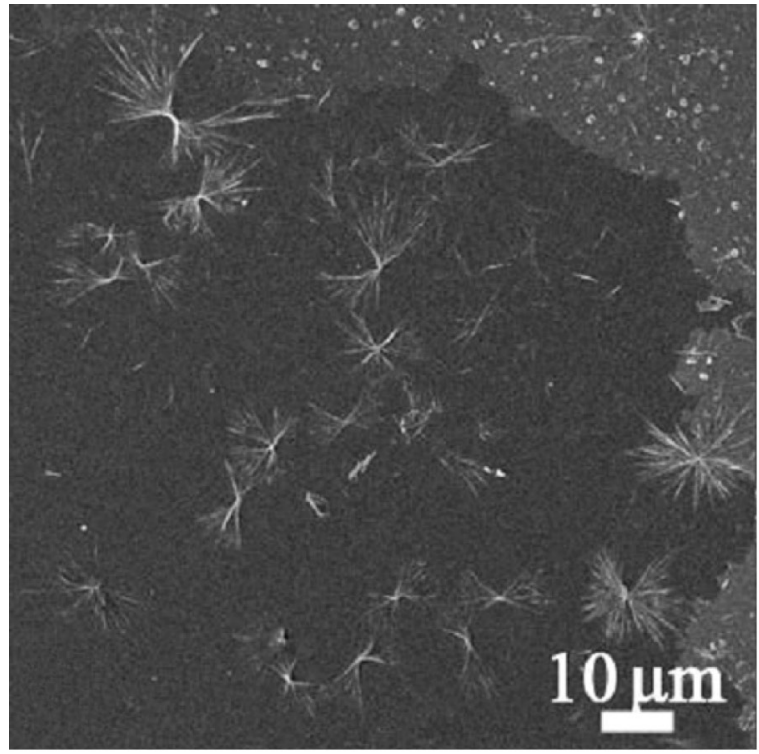

(a)

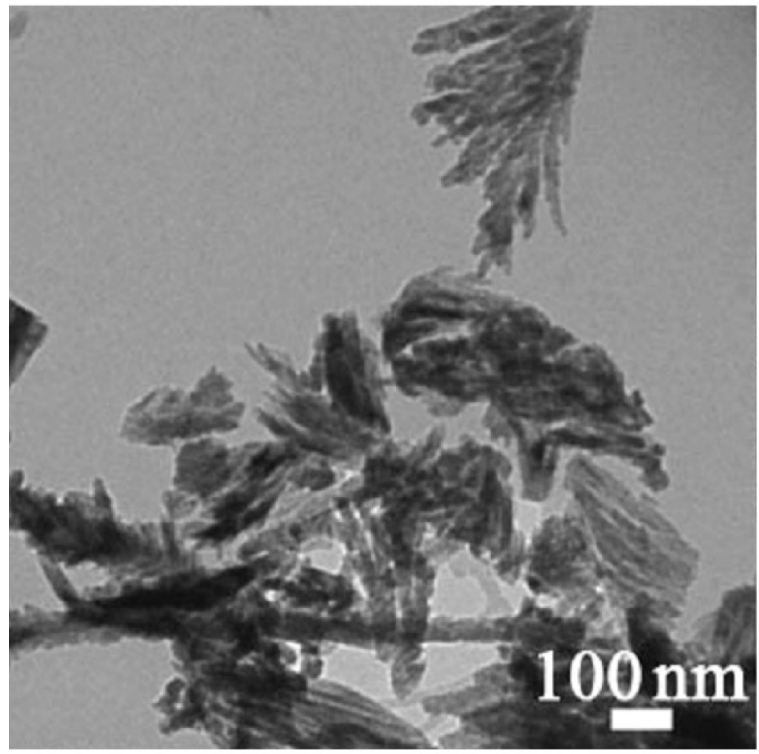

(c)

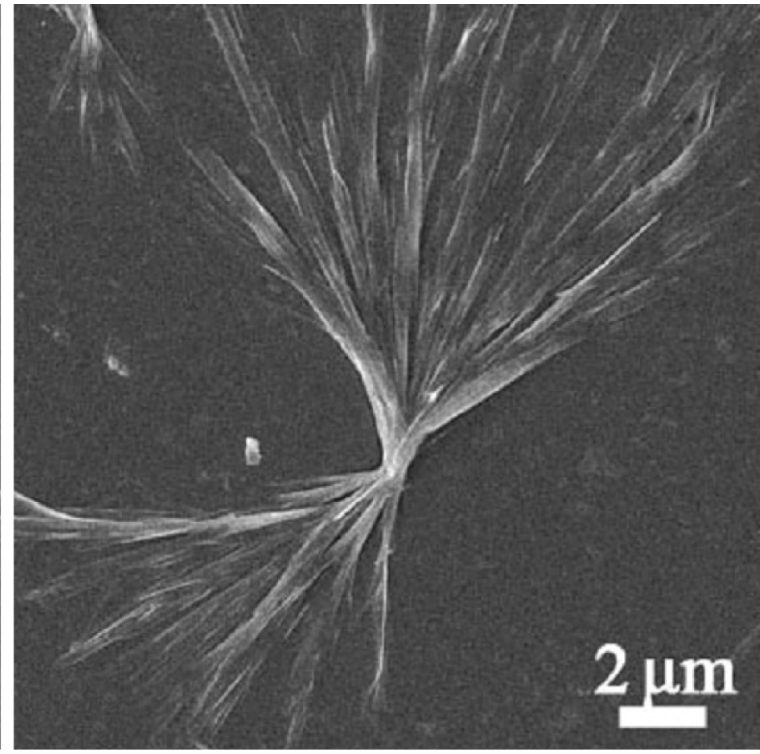

(b)

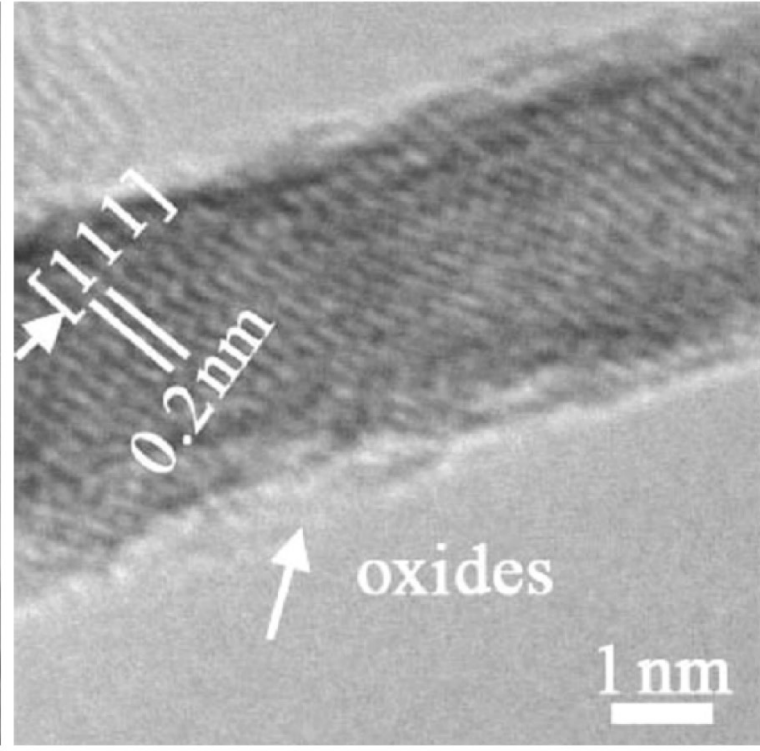

(d)

Figure 2. (a) SEM image and (b) high magnified SEM image of the products; (c) TEM image and (d) HRTEM image of the products.

resonance band may be interpreted as a consequence of size and morphology of the Ni architectures. Due to the fact that their lengths and widths are larger than a magnetic wall, the $\mathrm{Ni}$ architectures are made up of several magnetic domains so that the broad resonance band may be interpreted as a consequence of their magnetic polydomain configuration. The frequency band broadening is also related to the shape and morphology of the nanostructures because of the effect of the demagnetization fields ${ }^{15}$.

As a typical magnetic material, the magnetic loss of $\mathrm{Ni}$ architectures is mostly associated with magnetic hysteresis, domain wall resonance, eddy current loss, natural resonance, and exchange resonance for particles smaller than $100 \mathrm{~nm}^{[20,21]}$. Magnetic hysteresis stemming from irreversible magnetization occurs only in a highly applied field, whereas domain wall resonance derived from multi-domain materials occurs only in the sub-GHz frequency range. Exchange resonance should be excluded in the present system since the size of the $\mathrm{Ni}$ architectures is larger than $100 \mathrm{~nm}$. If the magnetic loss only stems from the eddy current loss, the values of $\mu^{\prime \prime}\left(\mu^{\prime}\right)^{-2} f^{-1}$ should be constant when the frequency is changed. As shown in Figure $3 \mathrm{~d}$, the values of $\mu^{\prime \prime}\left(\mu^{\prime}\right)^{-2} f^{-1}$ of the Ni architectures change remarkably with increasing frequency. Therefore, the magnetic loss in the present system is mainly caused by the natural resonance.

According to the transmission line theory, when a wave is normally incident to an absorber with a backed metal plate, the reflection loss (RL) curves at a given absorber 


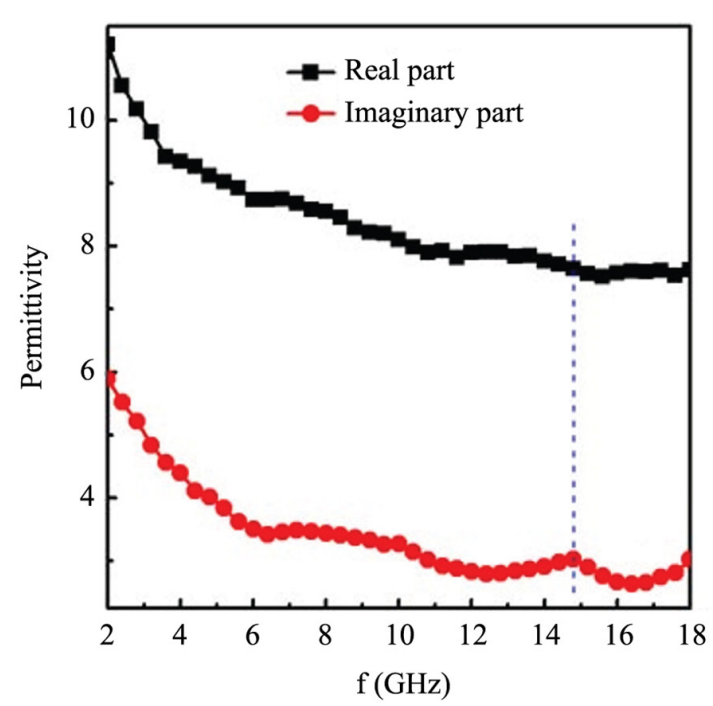

(a)

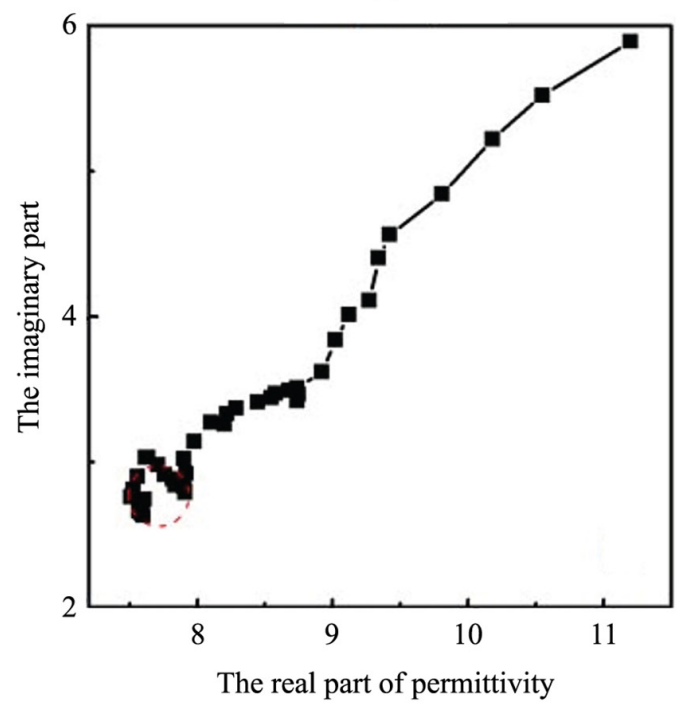

(c)

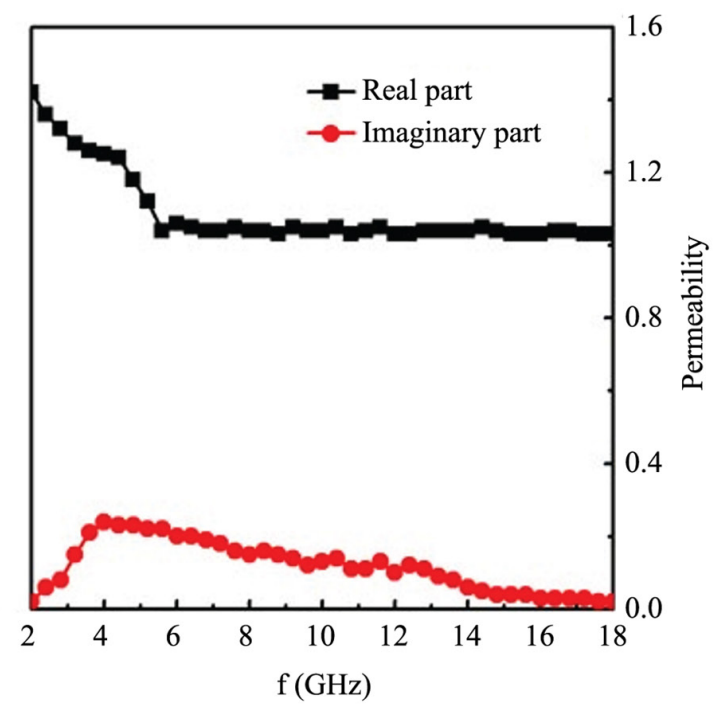

(b)

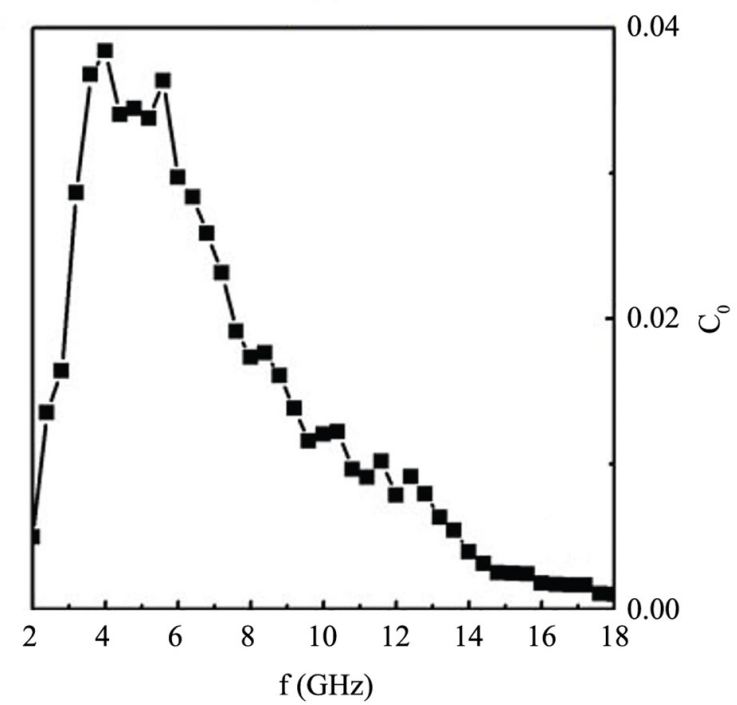

(d)

Figure 3. Frequency dependence of (a) the relative complex permittivity and (b) the relative complex permeability, (c) Cole-Cole plot, and (d) frequency dependence of $C_{0}\left(C_{0}=\mu^{\prime \prime}\left(\mu^{\prime}\right)^{-2} f^{-1}\right)$.

thickness can be calculated from the complex permeability and permittivity by means of the following expressions ${ }^{3,5-12}$ :

$$
\begin{aligned}
& Z=Z_{\text {in }} / Z_{0}=\sqrt{\mu_{r} / \varepsilon_{r}} \tanh \left((j 2 \pi t / \lambda) \sqrt{\mu_{r} \varepsilon_{r}}\right) \\
& R L=20 \lg |(Z-1) /(Z+1)|
\end{aligned}
$$

where $Z$ is the normalized input impedance related to the impedance in free space; $\lambda$ is the wavelength in free space; and $t$ is the thickness of the absorber.

The 3D plot of the RL of the paraffin-bonded $\mathrm{Ni}$ architectures composites against thickness $(1-5 \mathrm{~mm})$ and frequency $(2-18 \mathrm{GHz})$ is presented in Figure 4. It is clear that an optimal RL of $-38.9 \mathrm{~dB}$, corresponding to $99.97 \%$ absorption, is observed at $12.8 \mathrm{GHz}$ for a thickness of $2.1 \mathrm{~mm}$. By increasing the thickness, the RL maximum shifts to a lower frequency. When the thickness is thicker than the critical value, two peaks appear simultaneously. RL values exceeding $-20 \mathrm{~dB}$ are obtained in the $8.0-17.8 \mathrm{GHz}$ range with the thickness varying between 1.5 and $3.2 \mathrm{~mm}$. This frequency range covers the absorption frequency range of the traditional sintered ferrites ${ }^{22}$. The good microwave absorbing properties of $\mathrm{Ni}$ architectures can be ascribed to the excellent synergetic effect of the dielectric relaxation loss and the magnetic resonance loss.

A quarter-wavelength cancellation model has been successfully used to explain the relationship between RL peak frequency and absorber thickness for carbonyl-iron particles, $\mathrm{Ni} @ \mathrm{Ni}_{2} \mathrm{O}_{3}$ core-shell particles and $\mathrm{FeNi}_{3} / \mathrm{C}$ nanowires ${ }^{10,12,23}$. According to the model, the minimum RL can be gained at given frequencies if the absorber thickness $\left(t_{M}\right)$ satisfies:

$t_{m}=n c /\left(4 f_{m} \sqrt{\left|\varepsilon_{r} \mu_{r}\right|}\right)(\mathrm{n}=1,3,5 \ldots .$. 


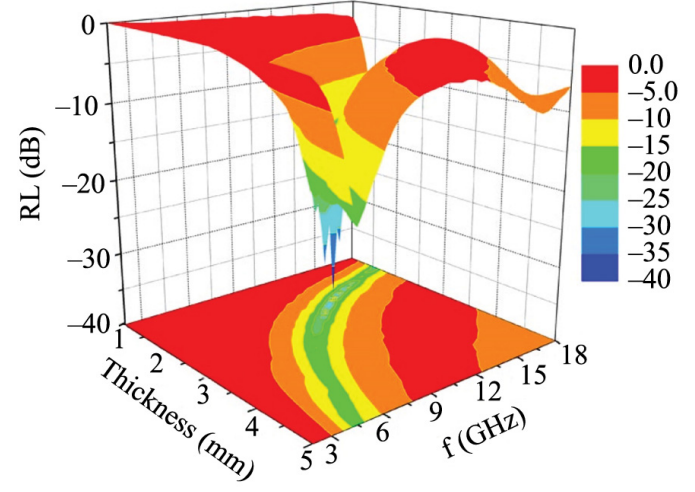

Figure 4. 3D representation of the RL derived from the measured and of the Ni-paraffin composites as a function of the frequency.

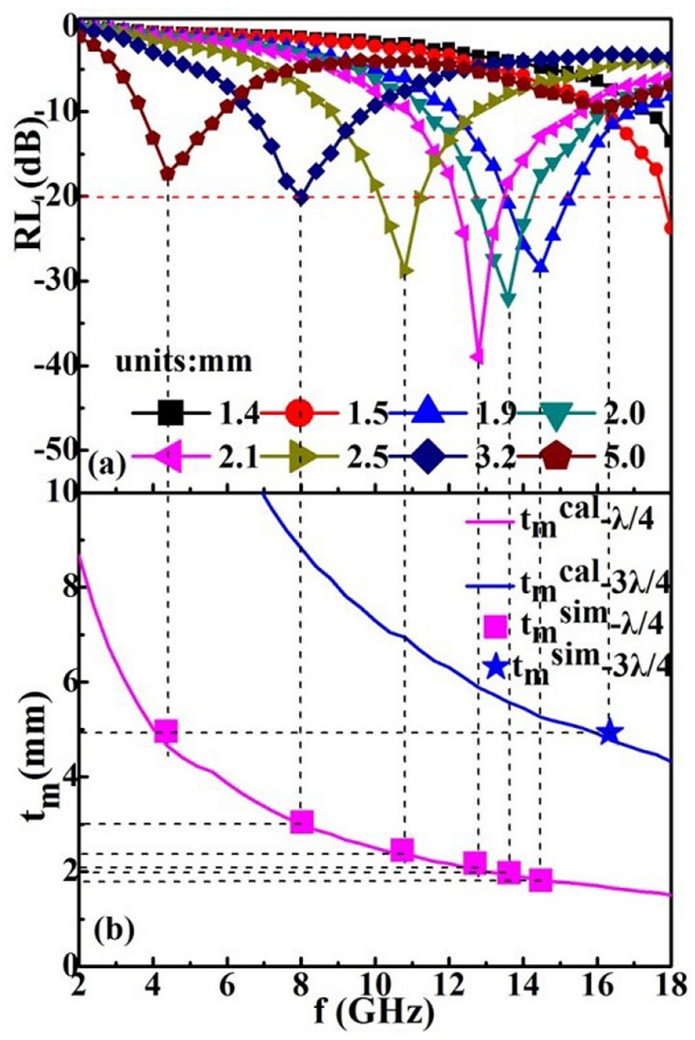

Figure 5. (a) Dependence of RL on frequency at various thicknesses for the Ni-paraffin composites; and (b) Dependence of $\lambda / 4$ and $3 \lambda / 4$ thickness on frequency for the Ni-paraffin composites.

\section{References}

1. Ohkoshi S, Kuroki S, Sakurai S, Matsumoto K, Sato K and Sasaki S. A millimeter-wave absorber based on gallium-substituted $\varepsilon$-iron oxide nanomagnets. Angewandte Chemie International Edition. 2007; 46(44):8392-8395. http://dx.doi.org/10.1002/ anie. 200703010 .

2. Namai A, Sakurai S, Nakajima M, Suemoto T, Matsumoto K, Goto M, et al. Synthesis of an electromagnetic wave absorber for high-speed wireless communication. Journal of the American Chemical Society. 2009; 131(3):1170-1173. http://dx.doi. org/10.1021/ja807943v. PMid:19115851. where $f_{M}$ is the peak frequency of RL, $\varepsilon_{r}$ and $\mu_{r}$ are the complex permittivity and permeability at $f_{M}$, respectively, and $c$ is the velocity of light.

Refering to Equation 2, the RL peak frequency is inversely proportional to the thickness, and two RL peaks appear at a sufficiently large thickness. The One at a lower frequency is relative to the $\lambda / 4$ condition, while the other one at a higher frequency comes from the $3 \lambda / 4$ condition. A comparison between the $t_{M}{ }^{C A L}$ calculated by Equation 2 (n equals 1 and 3 ) and the $t_{M}^{S I M}$ simulated by Equation 1 for the paraffin-bonded $\mathrm{Ni}$ architecture composites is shown in Figure 5. The good agreement between $t_{M}^{C A L}$ and $t_{M}{ }^{S I M}$ implies that the microwave absorption mechanism of the paraffin-bonded $\mathrm{Ni}$ architecture composites can be explained by the quarter-wavelength cancellation model ${ }^{10}$.

\section{Conclusions}

3D butterfly-like Ni architectures have been fabricated by a surfactant-assisted hydrothermal method. The as-prepared $\mathrm{Ni}$ architectures have been characterized by XRD, SEM, and TEM techniques and found to have a length of about $20 \mu \mathrm{m}$, a width of $4-6 \mu \mathrm{m}$, and be assembled by tens of Ni nanorods with a diameter of about $200 \mathrm{~nm}$. The $M_{S}$ of $\mathrm{Ni}$ architectures at $295 \mathrm{~K}$ has shown $66.2 \mathrm{emu} / \mathrm{g}$ and ferromagnetism. The EM properties of paraffin-bonded $\mathrm{Ni}$ architecture composites had been investigated. An absorber with a thickness of $2.1 \mathrm{~mm}$ has found to exhibit an optimal RL value of $-38.9 \mathrm{~dB}$ at $12.8 \mathrm{GHz}$. RL values exceeding $-20 \mathrm{~dB}$ have been obtained in the $8.0-17.8 \mathrm{GHz}$ range by choosing a thickness of 1.5-3.2 mm. The dielectric relaxation loss in the $\mathrm{Ni}$ architectures has been attributed to the interfacial relaxation between the oxide shells and the Ni nanorods as well as the size distribution and morphology of the Ni architectures, while the magnetic loss in the present system has been found to originate from the natural resonance. The thickness dependence on RL peak frequency has been described by a quarter-wavelength cancellation model. The present work suggests a great potential of using our 3D butterfly-like $\mathrm{Ni}$ architectures in microwave absorbing materials.

\section{Acknowledgements}

This study was supported by the National Natural Science Foundation of China (Grant No. 51201002), the Research Grants Council of the HKSAR Government (Grant No. PolyU 5236/12E), and The Hong Kong Polytechnic University (Grant Nos. G-YK59).

3. Ren YL, Wu HY, Lu MM, Chen YJ, Zhu CL, Gao P, et al Quaternary nanocomposites consisting of graphene, Fe3O4@ Fe core@shell, and ZnO nanoparticles: synthesis and excellent electromagnetic absorption properties. ACS Applied Materials \& Interfaces. 2012; 4(12):6436-6442. http://dx.doi.org/10.1021/ am3021697. PMid:23176086.

4. Watts PCP, Hsu WK, Barnes A and Chambers B. High Permittivity from defective multiwalled carbon nanotubes in the X-band. Advanced Materials. 2003; 15(7-8):600-603. http://dx.doi. org/10.1002/adma.200304485.

5. Zhang XF, Guan PF and Guo JJ. Dumbbell-like Fe3O4-Au nanoparticles for ultrabroadband electromagnetic losses by 
heterogeneous interfacial polarizations. Particle \& Particle Systems Characterization : Measurement and Description of Particle Properties and Behavior in Powders and Other Disperse Systems. 2013; 30(10):842-846. http://dx.doi.org/10.1002/ ppsc. 201300108.

6. Liu XG, Jiang JJ, Geng DY, Li BQ, Han Z, Liu W, et al. Dual nonlinear dielectric resonance and strong natural resonance in $\mathrm{Ni} / \mathrm{ZnO}$ nanocapsules. Applied Physics Letters. 2009; 94(5):053119. http://dx.doi.org/10.1063/1.3079393.

7. Yan SJ, Zhen L, Xu CY, Jiang YT and Shao WZ. Microwave absorption properties of $\mathrm{FeNi}_{3}$ submicrometre spheres and $\mathrm{SiO}_{2}$ @ $\mathrm{FeNi}_{3}$ core-shell structures. Journal of Physics. D, Applied Physics. 2010; 43(24):245003. http://dx.doi.org/10.1088/0022$3727 / 43 / 24 / 245003$.

8. Dong XL, Zhang XF, Huang $\mathrm{H}$ and Zuo F. Enhanced microwave absorption in Ni/polyaniline nanocomposites by dual dielectric relaxations. Applied Physics Letters. 2008; 92(1):013127. http:// dx.doi.org/10.1063/1.2830995.

9. Zhang XF, Dong XL, Huang H, Liu YY, Wang WN, Zhu XG, et al. Microwave absorption properties of the carbon-coated nickel nanocapsules. Applied Physics Letters. 2006; 89(5):053115. http://dx.doi.org/10.1063/1.2236965.

10. Wang BC, Zhang JL, Wang T, Qiao L and Li FS. Synthesis and enhanced microwave absorption properties of Ni@Ni2O3 core-shell particles. Journal of Alloys and Compounds. 2013; 567:21-25. http://dx.doi.org/10.1016/j.jallcom.2013.03.028.

11. Liu XG, Sun YP, Feng C, Jin CG and Li WH. Synthesis, magnetic and electromagnetic properties of $\mathrm{Al} 2 \mathrm{O} 3 / \mathrm{Fe}$ oxides composite-coated polyhedral Fe core-shell nanoparticles. Applied Surface Science. 2013; 280:132-137. http://dx.doi. org/10.1016/j.apsusc.2013.04.109.

12. Sun YP, Liu XG, Feng C, Fan JC, Lv YH, Wang YR, et al. A facile synthesis of FeNi3@C nanowires for electromagnetic wave absorber. Journal of Alloys and Compounds. 2014; 586:688-692. http://dx.doi.org/10.1016/j.jallcom.2013.10.063.

13. Wang ZZ, Zou JP, Ding ZH, Wu JF, Wang PH, Jin SW, et al. Magnetic and microwave absorption properties of Ni microcrystals with hierarchical branch-like and flowers-like shapes. Materials Chemistry and Physics. 2013; 142(1):119-123. http://dx.doi. org/10.1016/j.matchemphys.2013.07.003.

14. Song ZJ, Deng LJ, Xie JL, Zhou PH, Lu HP and Wang X. Synthesis, dielectric, and microwave absorption properties of flake carbonyl iron particles coated with nanostructure polymer.
Surface and Interface Analysis. 2014; 46(2):77-82. http://dx.doi. org/10.1002/sia.5351.

15. Wen SL, Liu Y, Zhao XC, Cheng JW and Li H. Synthesis, multinonlinear dielectric resonance and electromagnetic absorption properties of hcp-cobalt particles. Journal of Magnetism and Magnetic Materials. 2014; 354:7-11. http://dx.doi.org/10.1016/j. jmmm.2013.10.030

16. Wang H, Guo HH, Dai YY, Geng DY, Han Z, Li D, et al. Optimal electromagnetic-wave absorption by enhanced dipole polarization in Ni/C nanocapsules. Applied Physics Letters. 2012; 101(8):083116. http://dx.doi.org/10.1063/1.4747811.

17. Wu HJ, Wang LD, Wang YM, Guo SL and Shen ZY. Enhanced microwave performance of highly ordered mesoporous carbon coated by Ni2O3 nanoparticles. Journal of Alloys and Compounds. 2012; 525:82-86. http://dx.doi.org/10.1016/j. jallcom.2012.02.078.

18. Liu XG, Feng C, Or SW, Sun YP, Jin CG, Li WH et al. Investigation on microwave absorption properties of $\mathrm{CuO} /$ $\mathrm{Cu} 2 \mathrm{O}$-coated $\mathrm{Ni}$ nanocapsules as wide-band microwave absorbers. RSC Advances. 2013; 3(34):14590-14594. http:// dx.doi.org/10.1039/c3ra40937f.

19. Wang Y and Paulus B. A comparative electron correlation treatment in $\mathrm{H}(2) \mathrm{S}$-benzene dimer with DFT and wavefunctionbased ab initio methods. Chemical Physics Letters. 2007; 441(4-6):187-193. PMid:21072139.

20. Ma J, Li J, Ni X, Zhang XD and Huang JJ. Microwave resonance in Fe/SiO[sub 2] nanocomposite. Applied Physics Letters. 2009; 95(10):102505. http://dx.doi.org/10.1063/1.3224883.

21. Deng LJ, Zhou PH, Xie JL and Zhang L. Characterization and microwave resonance in nanocrystalline FeCoNi flake composite. Journal of Applied Physics. 2007; 101(10):103916. http://dx.doi.org/10.1063/1.2733610.

22. Wang H, Dai YY, Gong WJ, Geng DY, Ma S, Li D, et al. Broadband microwave absorption of CoNi@C nanocapsules enhanced by dual dielectric relaxation and multiple magnetic resonances. Applied Physics Letters. 2013; 102(22):223113. http://dx.doi.org/10.1063/1.4809675.

23. Wang T, Duan H, Lin X, Aguilar V, Mosqueda A and Zhao GM. Temperature dependence of magnetic anisotropy constant in iron chalcogenide $\mathrm{Fe}(3) \mathrm{Se}(4)$ : Excellent agreement with theories. Journal of Applied Physics. 2012; 112(10): 103905. http://dx.doi.org/10.1063/1.4767365. PMid:23258940. 